\title{
OSI Protocol Testing System based on User Friendly Test Scenario Specification Functions
}

\author{
Toshihiko Kato, Tomohiko Ogishi, \\ Toru Hasegawa and Kenji Suzuki
}

$K D D R \& D$ Laboratories

2-1-15, Ohara, Kamifukuoka-shi, Saitama 356, Japan

\begin{abstract}
As the OSI protocols come to be widely adopted in various communication systems, the testing of OSI protocol implementations becomes important. In this paper, we propose the OSI protocol testing system with user friendly test scenario specification functions aiming to support both the state transition oriented testing and the parameter oriented testing for the Transport through Application layer protocols. Our system introduces the separate tools with friendly human interface for specifying the test scenarios, for the test execution and for analyzing the test results. This paper describes the function and implementation of our system and shows its usefulness compared with the conventional testing system based on formal specification language.
\end{abstract}

Keyword Codes: C.2.2; D.2.5

Keywords: OSI Protocols; Conformance Testing

\section{Introduction}

As the standardization of OSI (Open Systems Interconnection) progresses, the OSI protocols come to be widely adopted in various communication systems. As a result, the testing of OSI protocol implementations becomes important in order to realize the interconnection between OSI based communication systems. A newly developed communication system will be tested, as testing during its development and/or conformance testing ${ }^{[1]}$, to ensure that it implements the relevant OSI protocol standards correctly. 
In such a testing, an OSI system needs to be tested for the behaviors of each layer protocol. The OSI protocols define the procedures to exchange PDUs (Protocol Data Units) as well as the data structure of the parameters conveyed in PDUs, and therefore an OSI system will be checked from the following testing aspects.

- State transition oriented testing : the testing that focuses on the procedures to exchange PDUs and service primitives modeled in a finite state machine.

- Parameter oriented testing : the testing that focuses on the values of the parameters of PDUs and service primitives.

Since the OSI protocols have rich functionality, the testing of an OSI system needs large number of test scenarios for those testing aspects.

In order to realize an effective testing of OSI systems, it is indispensable to establish the OSI protocol testing methods for supporting the test scenario description, the test execution according to given scenarios, and the test result analysis. Although several protocol testing methods for OSI systems have been proposed so far ${ }^{[2-6]}$, many of them focus only on the state transition oriented testing. Some of them support two testing aspects ${ }^{[5,6]}$. However, they are based on the test scenario specification using formal specification languages such as TTCN (Tree and Tabular Combined Notation) $)^{[1]}$ and PSL.1 ${ }^{[6]}$, and they have a problem that the efforts to specify test scenarios are laborious for the exhaustive testing of an OSI system.

This paper proposes a testing method supporting both the state transition oriented testing and the parameter oriented testing, which is based on the test scenario specification using a testing system with friendly human interface. The test operator will specify the sequence of the test events, for the state transition oriented testing, and the parameter values of a PDU and a service primitive corresponding to one test event, for the parameter oriented testing, with the help of our testing system. The mapping between the sequence and the parameter values of test events is maintained by our testing system. By introducing a testing system instead of a formal specification language, the efforts to specify test scenarios will be much smaller than the previous testing methods.

The next section describes the design principles for our testing system. Section 3 focuses on the human interface of our testing system for the test scenario editor, the test executor and the test result analyzer. Section 4 describes the implementation of our system. Section 5 discusses our system, and section 6 makes a conclusion on our work. 


\section{Design Principles of OSI Protocol Testing System}

We have adopted the following principles on the design of the OSI protocol testing system.

(1) Our system provides the testing capability for the OSI protocols from the Transport layer to the Application layer. By taking account of the independence of layers, our system adopts the layer by layer testing and provides the lower tester for the Transport layer through the Presentation layer and for ASEs (Application Service Elements) in the application layer. In order to eliminate the additional implementation of the upper tester in SUT (System Under Test), our system tests a protocol implementation (IUT : Implementation Under Test) as it is combined together with the higher layer implementation and application program in SUT. Figure 1 shows the testing scheme adopted in our system. Using the categorization defined in [1], our system uses the Remote Single-layer Embedded (RSE) test method.

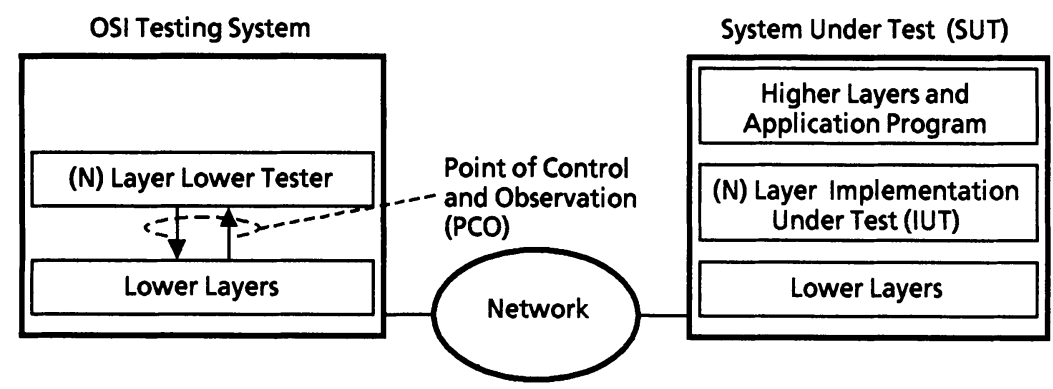

Figure 1 Testing Configuration

(2) In order to support the test scenario description, the test execution and the test result analysis, our testing system consists of the Test Scenario Editor, the Test Executor and the Test Result Analyzer. The Test Scenario Editor is a tool for describing and maintaining test scenarios and enables a new test scenario to be created both from the beginning and by modifying another scenario prepared previously. The Test Executor performs testing by interpreting test events described in a given test scenario and stores the results of execution in a test log. The Test Result Analyzer is a tool for analyzing test logs stored by the Test Executor.

(3) In order to support the state transition oriented testing and the parameter oriented testing, the Test Scenario Editor and the Test Result Analyzer have separate functionality for specifying and analyzing the test event sequence and the parameter values of a PDU and a service primitive. 
(4) The data structure of PDUs for the Presentation and Application layers is defined in ASN.1 (Abstract Syntax Notation One) ${ }^{[7]}$. We use the tool handling ASN.1 values, called AsTool/ $T^{[8]}$, to implement the PDU parameter value specification and analysis. By use of AsTool/T, any PDUs whose structure is defined in ASN.1 can be easily handled in our testing system.

(5) In order to support various Application layer protocols such as FTAM, MHS and CMIP, we adopt a generic framework that the testing capability for an Application protocol can be realized only by giving the PDU data structure described in ASN.1 and the mappings between PDUs and lower layer service primitives.

(6) Our testing system is implemented on a personal computer running Windows. As for the communication interface used by the Test Executor, we use the OSI 7 layer board which we have implemented ${ }^{[9]}$.

\section{Functions of OSI Protocol Testing System}

\subsection{Overview}

As described in section 2, our testing system consists of Test Scenario Editor, Test Executor, Test Result Analyzer. Based on the design principles in section 2, each tool will be further divided into several subtools. This subsection describes the overview of our OSI protocol testing system.

The Test Scenario Editor provides the following subtools for the test scenario specification.

- Test Event Sequence Editor which focuses on the state transition oriented testing and specifies the sequence of test inputs sent by the lower tester and expected responses received from SUT. It specifies the test inputs and expected responses at the point of control and observation (PCO) depicted in Figure 1.

- PDU Parameter Editor which focuses on the parameter oriented testing and supports to assign parameter values to PDUs used in the testing.

- Primitive Parameter Editor which also focuses on the parameter oriented testing and the assigning parameter values to lower layer's service primitives used at PCO below the lower tester.

The Test Executor sends a test input and waits for response from SUT, and shows the sent and received events on the display. It also stores the sent and received events in the test log for later offline analysis. 
The Test Result Analyzer consists of the following subtools.

- Test Event Sequence Analyzer which focuses on the state transition oriented testing and displays the sequence of test inputs and responses stored in the test log. As similar to the Test Event Sequence Editor, it gives the test event sequence at $\mathrm{PCO}$ below the lower tester.

- PDU Parameter Analyzer which focuses on the parameter oriented testing and shows all the parameter values contained in a sent or received PDU in a human readable form.

- Primitive Parameter Analyzer which also focuses on the parameter oriented testing and shows all the parameter values in a lower layer's service primitive used at PCO below the lower tester.

The following subsections describe the functions of these tools.

\subsection{Test Scenario Editor}

\subsubsection{Test Event Sequence Editor}

The Test Event Sequence Editor provides the functions summarized below in order to specify the sequence of test events, test inputs and responses, contained in a test scenario. Examples of the Test Event Sequence Editor, for MHS P1 and P2 protocols and the Session protocol, are depicted in Figure 2.

(1) It provides a window where test inputs to SUT and responses from SUT are specified. The test inputs and responses will be selected from the test event menu window listing all the test events allowed for the protocol being tested.

(2) In order to reduce the specification efforts, a test event is represented only by the name of PDU, or by the name of lower service primitive in case that the lower service primitive does not contain any PDU. The Test Event Sequence Editor maintains the mappings between PDUs and lower layer primitives, and the test operators do not need to specify the mappings in the test scenarios.

(3) The Test Event Sequence Editor provides the command to control the test event sequence, such as LOOP and WAIT. These commands are specified as test inputs.

(4) In order to specify the concatenation of PDU and the non-determinism in the receiving responses in the test event sequence, the Test Event Sequence Editor allows the supplementary commands to be specified together with test events. The followings give the functions of the supplementary commands. 


\begin{tabular}{|c|c|c|c|c|}
\hline \multicolumn{5}{|c|}{ Test Event Sequence Editor } \\
\hline \multicolumn{5}{|c|}{. } \\
\hline & \multicolumn{2}{|c|}{ Test Input } & \multicolumn{2}{|r|}{ Response } \\
\hline $\begin{array}{l}1 \\
2 \\
3 \\
4 \\
5 \\
6\end{array}$ & \multicolumn{2}{|c|}{$\begin{array}{l}\text { MTA Bind Argument } \\
\text { Message } \\
\text { RT-CLOSE req }\end{array}$} & \multicolumn{2}{|c|}{$\begin{array}{l}\text { MTA Bind Result } \\
\text { RT-TRANSFER conf } \\
\text { RT-CLOSE conf }\end{array}$} \\
\hline & PDU Editor & Primiti & itor & Save Scenario \\
\hline & Cut & & & Paste \\
\hline
\end{tabular}

\begin{tabular}{|c|c|}
\hline \multicolumn{2}{|c|}{ Test Event Menu } \\
\hline \multicolumn{2}{|c|}{\begin{tabular}{l} 
MTA Bind Argument \\
MTA Bind Result \\
MTA Bind Error \\
Message \\
Probe \\
Report \\
RT-TRANSFER resp \\
RT-TRANSFER conf \\
RT-CLOSE req \\
RT-CLOSE ind \\
RT-CLOSE resp \\
RT-CLOSE conf \\
RT-U-ABORT req \\
\multicolumn{1}{c}{ : } \\
\end{tabular}} \\
\hline \begin{tabular}{|l|l|l|}
+ & +1 & $?$ \\
\end{tabular} & \begin{tabular}{l|l|l}
$*$ & 1 & $>$ \\
\end{tabular} \\
\hline Input & Response \\
\hline Replace & \\
\hline
\end{tabular}

(a) Test Event Sequence Editor for MHS

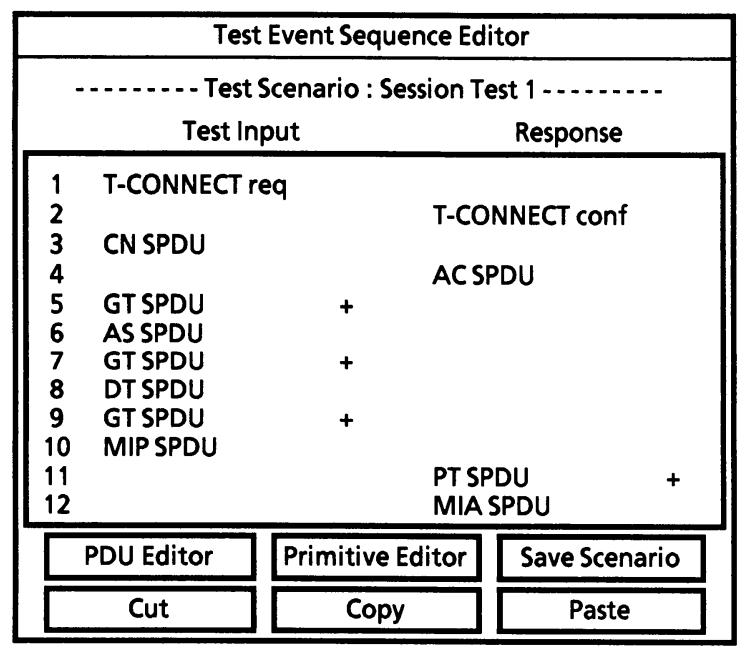

\begin{tabular}{|c|c|}
\hline \multicolumn{2}{|c|}{ Test Event Menu } \\
\hline \multicolumn{2}{|c|}{$\begin{array}{l}\text { CN SPDU } \\
\text { AC SPDU } \\
\text { OA SPDU } \\
\text { CDO SPDU } \\
\text { RF SPDU } \\
\text { FN SPDU } \\
\text { DN SPDU } \\
\text { NF SPDU } \\
\text { AB SPDU } \\
\text { AA SPDU } \\
\text { DT SPDU } \\
\text { EX SPDU } \\
\text { TD SPDU }\end{array}$} \\
\hline \begin{tabular}{l|l|l}
+ & +1 & $?$
\end{tabular} & \begin{tabular}{|l|l|l|}
$*$ & 1 & $>$ \\
\end{tabular} \\
\hline Input & Response \\
\hline Replace & Insert \\
\hline
\end{tabular}

(b) Test Event Sequence Editor for Session Protocol

Figure 2 Human Interface of Test Event Sequence Editor

- "+" indicates that the PDUs combined by "+" are concatenated and transferred as a user data of one lower layer service primitive.

- " + " indicates that several PDUs of the same type may be concatenated in a received lower layer primitive. 
- "?" indicates that the response may not be received.

- "**" indicates that the response may be received repeatedly.

- " " indicates that the responses combined by " "may be received in any order.

- ">" indicates that the parameters for the test input are not specified completely. For the test input, the Test Executor will be suspended and allow the parameters to be specified during the test execution phase.

(5) In order to facilitate the sequence specification, the Test Event Sequence Editor provides some editing functions for the test event sequence, such as copy, cut and paste commands.

(6) The Test Event Sequence Editor will invoke the PDU Parameter Editor or the Primitive Parameter Editor for a test event, when the PDU Editor button or the Primitive Editor button is pushed by the test operator, respectively.

\subsubsection{PDU Parameter Editor}

Our testing system provides different forms of PDU Parameter Editor for the Presentation and Application layers, and for the Transport and Session layers.

As for the Presentation and Application layers, the PDUs has a tree structure defined by ASN.1. So, the PDU Parameter Editors for those layers have the tree based human interface, which is provided by AsTool/T. The test operator can read and modify a particular element after identifying the element by selecting the path from the root element to the corresponding element in the tree of the PDU structure. Figure 3 depicts an example of human interface of the PDU Parameter Editor for MHS P1 and P2 protocols. The functions of the editor are summarized as follows :

(1) A display of the editor represents a parameter element in a PDU. The display format contains

- the reference name of the element, the encoding identifier which contains class, primitive or constructor form and ID code, and the type name of the element,

- the reference name and the related information of the subelements which are included in this elements, and

- the command line from the operator.

(2) The test operator traverses the tree of the PDU elements downward by selecting the number of the subelement in the parent element (see Figure 3 (a) and (b)), and upward by typing a carriage return in the subelement's display. 


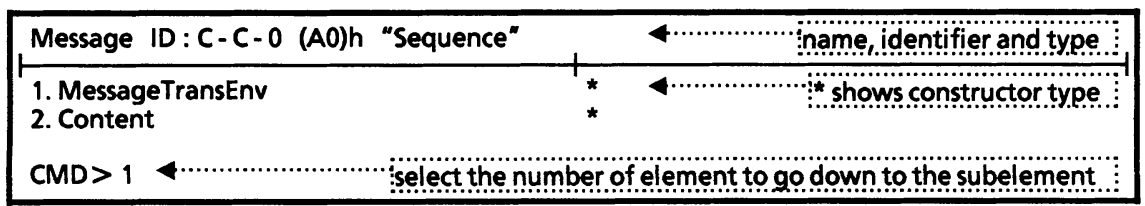

(a) Message MTSapdu

\begin{tabular}{|c|c|}
\hline MessageTransEnv ID : U - C - 17 (31)h "Set" & \\
\hline $\begin{array}{l}\text { 1. Messageld } \\
\text { 2. OriginatorName } \\
\text { 3. OrgEncodedlnfType } \\
\text { 4. ContentType } \\
\text { 5. Contentld } \\
\text { 10. MessageReciplnfo } \\
\text { 11. Tracelnfo }\end{array}$ & 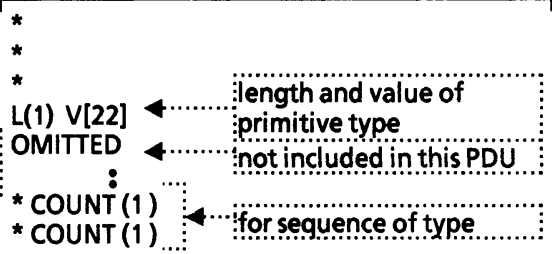 \\
\hline
\end{tabular}

(b) Message Transfer Envelope

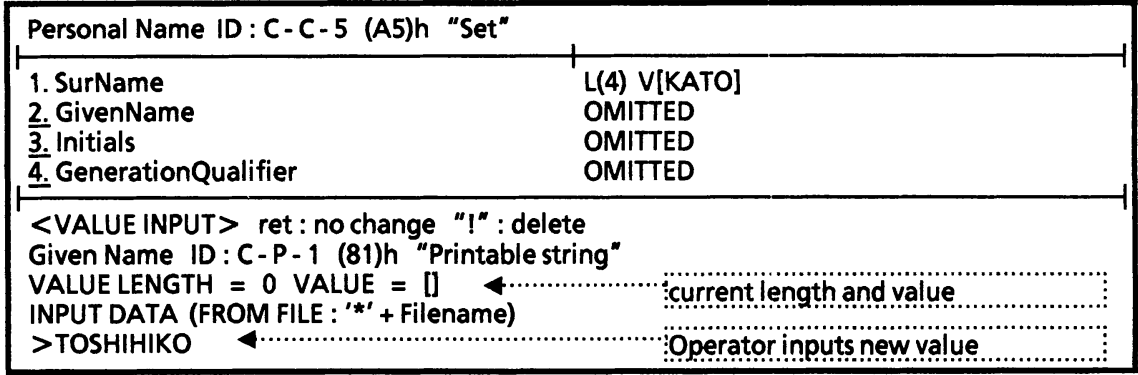

(c) Modifying the value for Personal Name

Figure 3 Human Interface of PDU Parameter Editor for MHS

(3) The test operator modifies the value of the elements by selecting the primitive elements. Figure 3 (c) shows the modification of the "GivenName" in the "PersonalName" of the originator's O/R name.

On the other hand, our testing system provides the PDU Parameter Editor with table based human interface for the Transport and Session layers. Figure 3 shows an example of human interface of the PDU Parameter Editor for the Session protocol. The functions of the editor are summarized as follows :

(1) The editor provides a table for each PDU. The table contains all the parameters defined for the PDU.

(2) The test operator selects a parameter and sets the value in the input column (see Figure 4). In case that any values may be assigned for the parameter, the editor allows the test operator to input any ASCII or octet string in the input 


\begin{tabular}{|c|c|c|c|c|c|}
\hline \multicolumn{6}{|c|}{ CONNECT SPDU } \\
\hline & & & & & Input columnn \\
\hline PGI & $\mathrm{m} / \mathrm{nm}$ & $\mathbf{P I}$ & $\mathrm{m} / \mathrm{nm}$ & Length & Parameter Value \\
\hline \multirow{3}{*}{$\begin{array}{l}\text { Connection } \\
\text { identifier }\end{array}$} & \multirow{3}{*}{$\mathrm{nm}$} & Calling SS-user ref & $\mathrm{nm}$ & 7 & KDDPSAP \\
\hline & & Common ref & $\mathrm{nm}$ & 17 & $199404011312+0900$ \\
\hline & & Additional ref info & $\mathrm{nm}$ & 0 & (not used) \\
\hline $\begin{array}{l}\text { Connect / } \\
\text { Accept item }\end{array}$ & $\mathrm{nm}$ & $\vdots$ & $\vdots$ & $\vdots$ & $\vdots$ \\
\hline$:$ & : & : & : & : & : \\
\hline User data & $\mathrm{nm}$ & & & 100 & rtorq.pdu 1100 \\
\hline
\end{tabular}

Figure 4 Human Interface of PDU Parameter Editor for Session Protocol

column. In case that only the defined values are assigned to the parameter, the editor prepares the value menu for the parameter.

(3) The user data parameter needs to contain the higher layer PDU. The editor assigns the user data parameter in the following way.

- The test operator encodes the higher layer PDU using the PDU Parameter Editor of the higher layer.

- When the user data parameter is selected, the editor shows a window for selecting the file containing the higher layer PDU and for indicating the offset in the file and the length for the data which is contained in the user data parameter. By giving the offset and the length, it is possible to segment the higher layer PDU into the user data parameters of more than one PDUs.

\subsubsection{Primitive Parameter Editor}

Our testing system prepares the Primitive Parameter Editor for the Network through Presentation layers and the commonly used ASE such as ACSE, RTSE and ROSE. Figure 5 gives an example of human interface of the Primitive Parameter Editor for RTSE.

\subsection{Test Executor}

The Test Executor reads a test scenario and interprets the test events described in the scenario. It sends the specified PDU and /or service primitive when the test event is a test input. When the test event is a response, it waits for receiving a PDU and /or service primitive and checks whether the received PDU and / or service primitive are identical to those specified in the test scenario. The Test Executor shows the test inputs sent and the responses received on a window 


\begin{tabular}{||c|c|c|l||}
\hline \multicolumn{4}{|c|}{ RT-OPEN req } \\
\hline \multicolumn{3}{|c|}{} \\
\hline Parameter & Subparameter & Length & \multicolumn{3}{|c|}{ Parameter Value } \\
\hline Dialogue-mode & & 2 & two-way-alternate \\
\hline Initial-turn & & 2 & Initiator side \\
\hline \multirow{3}{*}{$\begin{array}{c}\text { Calling } \\
\text { presentation } \\
\text { address }\end{array}$} & P Selector & 7 & KDDPSAP \\
\cline { 2 - 5 } & S Selector & 7 & KDDSSAP \\
\cline { 2 - 5 } & T Selector & 7 & KDDTSAP \\
\cline { 2 - 5 } & N Address & 9 & 44082XXX............... \\
\hline
\end{tabular}

Figure 5 Human Interface of Primitive Parameter Editor for MHS

together with the time stamp. It also stores in the test log the data actually transferred between the lower tester and IUT.

When the supplementary command " $>$ " is specified with a test input, the Test Executor stops and invokes the Test Scenario Editor and the Test Result Analyzer in order to set parameter values for the test input. This allows a parameter in a test input to be determined during the test execution, for example, in response to a response received before the test input.

\subsection{Test Result Analyzer}

The Test Result Analyzer has a similar structure with the Test Scenario Editor. The Test Event Sequence Analyzer shows the sequence of the actually transferred test inputs and responses using the information stored in the test log.

By selecting a test event displayed by the Test Event Sequence Analyzer and invoking the PDU Parameter Analyzer, it lists up all the parameter values contained in the PDU. For the Presentation and Application layers, the PDU Parameter Analyzer is based on AsTool/T and gives information on the type and value of the elements with representing the tree structure of the PDU definition. Figure 6 shows an example of human interface of the PDU Parameter Analyzer for MHS. The PDU Parameter Analyzer for the Transport and Session layers lists up the names and the values of parameters contained.

Similarly, the Primitive Parameter Analyzer is invoked from the Test Event Sequence Analyzer for the selected test event. It lists up the names and the values of parameters contained in the service primitive corresponding to the test event. 


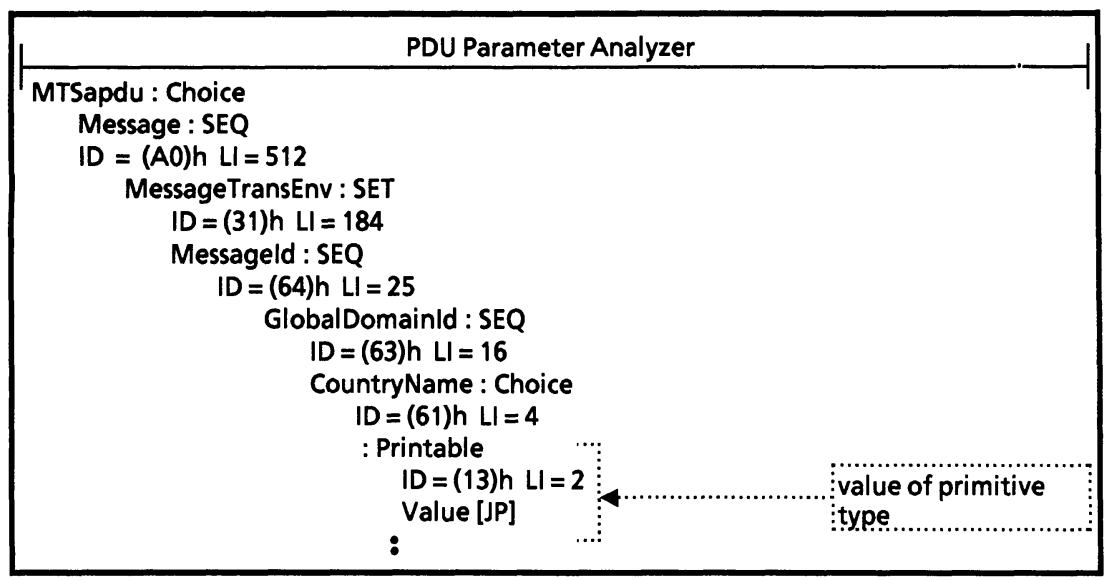

Figure 6 Human Interface of PDU Parameter Analyzer for MHS

\section{Implementation of OSI Protocol Testing System}

The key issue to implement our system is how to realize the generic framework for supporting various Application layer protocols. Our system have adopted the following approach.

(1) For an Application layer protocol, the definition of PDU structure in ASN.1 and the mappings between PDUs and lower layer service primitives are given to the testing system (see Figure 7). The ASN.1 definition of PDUs is applied to AsTool/T ASN.1 parser and is converted into PDU structure data.

(2) The generic program for the Test Event Sequence Editor reads the mappings between PDUs and lower layer service primitives, and gets knowledge on what kind of PDUs are used and what service primitives are used for conveying the PDUs. Based on the knowledge, the generic program realizes the Test Event Sequence Editor for the Application layer protocol.

(3) As for the PDU Parameter Editor, our system has TPDU and SPDU Parameter Editors and the AsTool/T PDU Editor (see Figure 7). The PDU Parameter Editor for the Application and Presentation layers is realized by applying the PDU structure data to the AsTool/T PDU Editor.

(4) Our system has installed the Primitive Parameter Editor for the Network through Presentation layers, ACSE, ROSE and RTSE. The mappings between PDUs and lower layer service primitives are used to decide which primitive is used for an individual PDU (see Figure 7). 


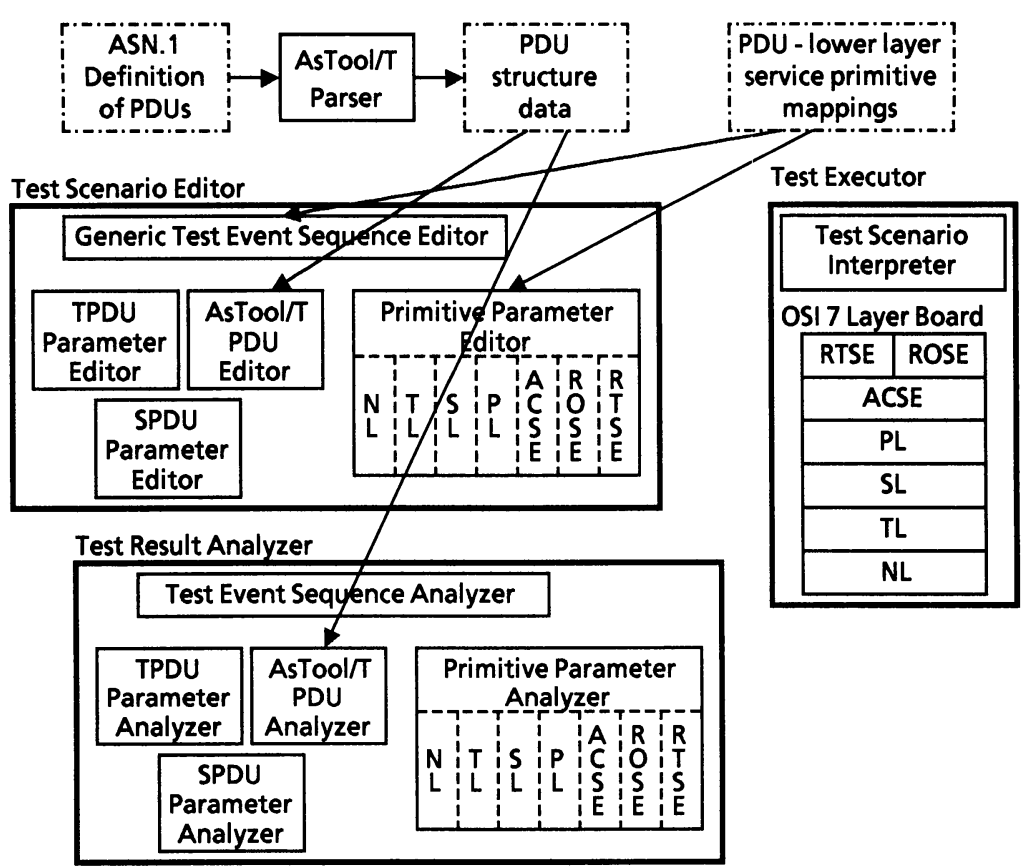

Figure 7 Overall Structure of OSI Protocol Testing System

(5) As for the Test Executor, the Test Scenario Interpreter performs the testing according to a given test scenario. The Test Scenario Interpreter sends and receives PDUs by exchanging service primitives through the OSI 7 layer board ${ }^{[9]}$ implementing the lower layer protocols (see Figure 7). The test scenario includes sufficient information to decide the lower layer service primitive conveying a PDU.

(6) The PDU structure data are applied to the AsTool/T PDU Analyzer in order to realize the the PDU Parameter Analyzer for the Application layer protocol.

\section{Discussions}

(1) Our testing system provides the Test Event Sequence Editor to describe the test scenario from the standpoint of the state transition oriented testing. The editor allows the test operator to describe the test event sequence using only the names of PDUs or lower layer service primitives. This makes the description much simpler than the conventional testing method.

Figure 8 shows an example of TTCN description of the same test event sequence as that depicted in Figure 2 (a). In the TTCN description, the PCO name 
(" $L$ " in this case), the name of lower service primitives, and the parameter names and values of the service primitives need to be specified for every test event. The TTCN description also requires to specify the default cases in the sequence (" $L$ ? OTHERWISE" and "L ? TIMEOUT" in this case).

\begin{tabular}{|c|c|}
\hline \multicolumn{2}{|l|}{ Dynamic Behaviour } \\
\hline Behaviour Description & Verdict \\
\hline $\begin{array}{l}\text { L! RT-OPEN req [Dialogue-mode } \sim \text { TWA, } \cdots \text {, User-data } \sim \text { MTA Bind Argument] } \\
\text { L ? RT-OPEN conf [User-data } \sim \text { MTA Bind Result] } \\
\text { L! RT-TRANSFER req [Transfer-time } \sim 10000, \text { APDU } \sim \text { Message] } \\
\text { L? RT-TRANSFER conf } \\
\text { L! RT-CLOSE req } \\
\text { L ? RT-CLOSE conf } \\
\text { L ? OTHERWISE } \\
\text { L? TIMEOUT } \\
\text { L ? OTHERWISE } \\
\text { L? TIMEOUT } \\
\text { L? OTHERWISE } \\
\text { L? TIMEOUT }\end{array}$ & $\begin{array}{l}\text { pass } \\
\text { fail } \\
\text { fail } \\
\text { fail } \\
\text { fail } \\
\text { fail } \\
\text { fail }\end{array}$ \\
\hline
\end{tabular}

Figure 8 Description of Test Event Sequence with TTCN

On the other hand, the Test Event Sequence Editor in our system use only the names of PDUs (or lower service primitive if no PDU is used) because of the following reasons.

- Since the only PCO used in the Remote Single-layer Embedded test method we adopted is that below the lower tester, it is not necessary to specify the PCO name explicitly.

- Since our system maintains the mappings between PDUs and lower service primitives, the names of service primitives do not need to be specified together with PDUs.

- We have introduced another editor to specify the parameter values of primitives.

- We have introduced the semantics that the test event sequence not described is an incorrect sequence, and have eliminated the specification of the default cases.

The comparison between Figure 2 (a) and Figure 8 shows the simplicity of our description.

(2) Our testing system has adopted the tree based human interface in the PDU Parameter Editor for the Presentation and Application layers. This interface is contrast with other testing systems based on formal specification languages ${ }^{[5,6]}$. Figure 9 shows an example of the PDU parameter value in ASN.1 value 
description ${ }^{[7]}$ which is adopted by TTCN. This example corresponds to the parameter value depicted in Figure 3.

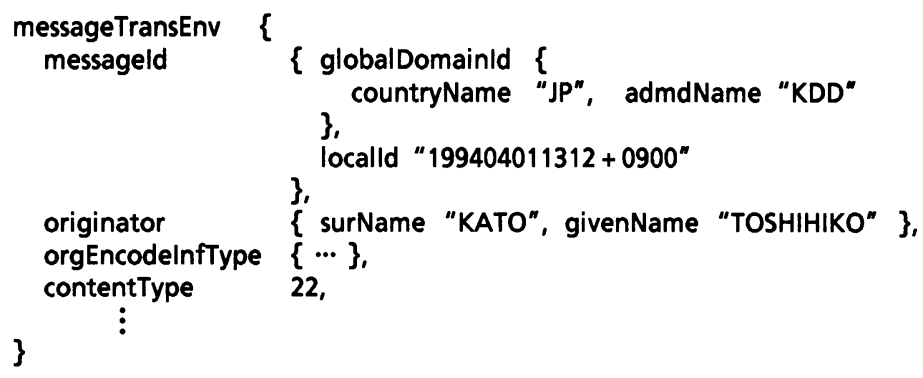

Figure 9 Description of PDU Parameter in ASN.1 value description

Comparing this style of format with our system, our interface is more appropriate in the following points.

- The standard ASN.1 value description requires all the elements in PDU to be described in one specification. On the other hand, our interface allows the individual elements to be focused on. Therefore, our system will make the editing of PDU with a complicated structure and with a lot of elements much easier than the testing system based on the ASN.1 value description .

- Our interface give the information on the type of elements, and therefore it allows the testing operator to understand much more easily the structure of the PDU and the correspondence between the value and the type.

- The method using the ASN.1 value description is apt to include the errors both syntactically, e.g. the mismatch of the parentheses, and semantically, e.g. the missing of the mandatory parameters.

(3) By preparing the separate tools for describing and analyzing the test event sequence, the PDU parameters and the primitive parameters, the reuse of the test scenarios becomes easy. The test event sequence and the parameters of PDUs and primitives can be reused independently. For example, the PDU Parameter Editor allows the test operator to specify the parameter of a PDU using any PDU parameters in other test scenarios independently designed, and using parameters of received PDUs stored when other test scenarios were executed previously.

Currently our system uses the conventional file systems in personal computers to store the test scenarios, the PDU and primitive parameters, and the test logs. In order to facilitate the retrieval of the stored test event sequence and parameters, the browser for the test scenario and test log may be useful. 
(4) With the help of the software tool handling ASN.1 values, AsTool/T, and the generic framework described in section 5, our testing system can easily support various Application protocols. So far, we have implemented the testing system for MHS P1 and P2 protocols. We believe that the testing system for other protocols such as FTAM and CMIP can be developed easily by use of our system.

\section{Conclusion}

In this paper, we have described the OSI protocol testing system with user friendly test scenario specification functions. Our testing system aims to support both the state transition oriented testing and the parameter oriented testing for the Transport through Application layer protocols. It introduces the separate tools for specifying the test scenarios, for the test execution and for analyzing the test results.

The test scenario specification tool, the Test Scenario Editor, includes the following subtools. The Test Event Sequence Editor specifies the sequence of the test events using only the names of PDUs or service primitives. The parameters of PDUs and service primitives are specified with the support of the PDU Parameter Editor and the Primitive Parameter Editor respectively. The introduction of separate subtools for the state transition oriented testing and the parameter oriented testing allows the test operator to specify the test scenarios much easily than the conventional testing systems based on formal specification languages such as TTCN.

The Test Executor performs the testing based on a given test scenario. It stores the test inputs and responses transferred between the testing system and the system under test. The Test Result Analyzer supports the offline analysis of the testing. It supports the state transition oriented testing and the parameter oriented testing using the subtools, the Test Event Sequence Analyzer, the PDU Parameter Analyzer and the Primitive Parameter Analyzer.

So far, we have implemented the testing function for the Transport and Session protocols and the MHS P1 and P2 protocols in our system. This system is being used for the testing of MHS systems developed at KDD. We are going to implement the testing system for other OSI protocols such as FTAM and CMIP based on the similar approach. 


\section{Acknowledgment}

The authors wish to thank to Dr. K. Urano, Director, and Mr. K. Maya, Deputy Director of KDD R \& D Laboratories, for their continuous encouragement of this study.

\section{References}

[1] CCITT, "Recommendation X.290 - OSI Conformance Testing Methodology and Framework for Protocol Recommendation for CCITT Applications," 1988.

[2] B. Sarikaya, G. Bochmann and E. Cerny, "A Test Design Methodology for Protocol Testing," IEEE Trans. Software Eng. Vol. SE-13 No. 5, pp.518 - 531, 1987.

[3] T. Kato, K. Suzuki and Y. Urano, "Conformance Testing for OSI Protocols in the Multiple Layer Environment based on Automaton Model," in Proceedings of ICCC '86, 1986.

[4] K. Takahashi, S. Nitanai, K. Gotoh, Y. Ishihata and K. Takahashi, "Design and Implementation for the Conformance Test System ACTS (in Japanese)," IPS Japan SIG Reports, DPS-59-2, 1993.

[5] S. Eswara, T. Berriman, P. VanHoutte and B. Sarikaya, "Towards Execution of TTCN Test Cases," in Protocol Specification, Testing and Verification X, pp. 99$112,1990$.

[6] H. Tsuji, F. Sato, K. Katsuyama, T. Mizuno and M. Soga, "Test Data Generation for Communication Software and Its Testing Method by Formal Approach (in Japanese)," Trans. of IPS Japan, Vol. 34, No. 6, pp. 1347-1360, 1993.

[7] CCITT, "Recommendation X.208 - Specification of Abstract Syntax Notation One (ASN.1)," 1988.

[8] T. Hasegawa, S. Nomura and H. Horiuchi, "Development of Software Tools for ASN.1 - Compiler and Editor - (in Japanese)," IPS Japan SIG Reports, DPS-394, 1988.

[9] A. Idoue, T. Kato and K. Suzuki, "Implementation and Evaluation of OSI 7 Layer Board (in Japanese)," IPS Japan SIG Reports, DPS-61-28, 1993. 